\title{
An algorithm for sofic shift equivalence*
}

\author{
K. H. KIM AND F. W. ROUSH \\ Mathematics Research Group, Alabama State University, Montgomery, \\ Alabama 36195, USA
}

(Received 20 January 1987 and revised 13 July 1987)

\begin{abstract}
We prove the decidability of shift equivalence of sofic systems and discuss algebraic invariants.
\end{abstract}

\section{Introduction}

Nasu [5], Nasu and Hamachi [3] showed that conjugacy of sofic shifts and resolving maps can be expressed in terms of strong shift equivalence with matrices of noncommuting variables. This extends the fundamental result of Williams to the sofic case. Boyle and Krieger [1] building on their work showed that strong shift equivalence can be conveniently handled as strong shift equivalence in a reduced semigroup semiring $\mathbb{Z}_{0}^{+}\left(M_{n}\left(\mathbb{Z}^{+}\right)\right)$. They defined shift equivalence of sofic systems and proved it is equivalent to eventual conjugacy.

As usual a subshift of finite type is the subset of $N^{\mathbf{Z}}=\left\{\left(a_{i}\right)\right\}, N=\{1,2, \ldots, n\}$ such that for all $i$ the $a_{i}, a_{i+1}$ entry of a given matrix $A$ is nonzero, and the shift $\mathscr{S}$ changes each coordinate to the next. All subshifts are topologized as subsets of the product of discrete topologies on $N^{\mathbf{Z}}$. A subshift is a general closed shift invariant subset of $N^{7}$.

Equivalently instead of a set of points $\left(a_{i}\right)$ subshift can be considered as the isomorphic sequence of arcs $\left(a_{i} a_{i+1}\right)$ in the graph of $\boldsymbol{A}$.

Definition. Let $\mathscr{S}_{1}, \mathscr{S}_{2}$ be subshifts of $N^{\mathbf{Z}}, M^{\mathrm{Z}}, N=\{1,2, \ldots, n\}, M=\{1,2, \ldots, m\}$ and $\psi$ a map $N \rightarrow M$ which induces a map $\mathscr{S}_{1}$ to $\mathscr{Y}_{2}$. It is right resolving if whenever $a_{1} a_{2}$ and $a_{1} a_{3}$ occur as two element subsequences (two blocks) of members of $\mathscr{S}_{1}$ and $\psi\left(a_{2}\right)=\psi\left(a_{3}\right)$ then $a_{2}=a_{3}$. Left resolving is the same condition for $a_{2} a_{1}, a_{3} a_{1}$. Biresolving means left and right resolving.

Definition. The $m$ th power of a subshift $\mathscr{S}$ of $N^{\mathbf{Z}}$ is the subshift of $\left(N^{m}\right)^{\mathbf{Z}}$ induced by the $m$ th power of the shift map whose underlying set is the image of the original under the map which sends $\left(a_{k}\right)$ to $\left(\left(a_{m k}, a_{m k+1}, \ldots, a_{m k+m-1}\right)\right)$.

Definition. Two subshifts are eventually conjugate if and only if there exist conjugacies of their $m$ th powers for all $m \geq m_{0}$ for some $m_{0}$.

Definition. Two maps $h: Z \rightarrow W, f: X \rightarrow Y$ are conjugate if there exist conjugacies $c: Z \rightarrow X, d: W \rightarrow Y$ such that $d h=f c$. 
Two resolving maps are eventually conjugate if and only if the induced maps $Z^{n} \rightarrow Y^{n}, X^{n} \rightarrow Y^{n}$ are conjugate for all sufficiently large $n$.

Kitchens and Nasu independently characterized maps topologically equivalent to resolving maps and gave an algorithm for recoding such maps as resolving maps.

Nasu [5] defined a $\lambda$-matrix as a matrix whose entries are formal sums

$$
\sum_{a \in A} k(a) a, \quad k(a) \in \mathbb{Z}^{+}, \quad a \in A_{0},
$$

where $A_{0}$ is an alphabet.

Any map of subshifts of finite type $A$ to $B$ can be represented up to conjugacy as a map of sequences $\left(a_{i} a_{i+1}\right)$ induced by a map $f$ from the edges of the graph of a matrix for $\boldsymbol{A}$ into the edges of the graph of a matrix for $B$. We obtain a $\lambda$-matrix by taking the matrix of the graph for $A$, and labelling nonzero entry corresponding to an edge $\alpha$ of the graph of $A$, by the label $f(\alpha)$. These $\lambda$-matrices are in general not unique.

TheOREM (Nasu [5] and Hamachi and Nasu [3]). Two factor maps are conjugate if and only if the $\lambda$-matrices are strong shift equivalent.

\section{Representations}

Definition. The semigroup semiring $\mathfrak{R}(\widetilde{S})$ for a semigroup $\subseteq$ over a semiring $\mathfrak{M}$ is the set of functions $f: \subseteq \rightarrow \Re$ which are zero for all but a finite number of elements of $\mathfrak{S}$, added by $f(x)+g(x)$ and multiplied by $f g(z)=\sum_{x y=z} f(x) g(y)$.

Definition. A representation of a semigroup (semiring) $\subseteq$ into a semiring $\mathfrak{A}$ is a multiplicative (multiplicative and additive) homomorphism of $\widetilde{S}$ into $\mathfrak{R}$.

Definition. A left module over a semiring $\Re$ with 0,1 is an additive commutative semigroup $\mathfrak{\Re}$ with 0 and a multiplication $\mathfrak{A} \times \mathfrak{K} \rightarrow \mathfrak{I}$ such that for all $a, b \in \mathfrak{A}, d, e \in \mathfrak{M}$ :

$$
\begin{array}{lll}
d(a+b)=d a+d b, & (d+e) a=d a+e a, & d(e a)=(d e) a \\
1 a=a, & 0 a=0, & d 0=0 .
\end{array}
$$

Definition. A left action of a semigroup $\subseteq$ on a module $\mathfrak{M}$ is a product $\subseteq \times \mathfrak{M} \rightarrow \mathfrak{M}$ preserving module operations such that

$$
d(e(a))=(d e) a \text { for } d, e \in \subseteq, a \in \mathfrak{M} .
$$

Right actions and modules are defined by reversing products in the above.

The right regular representation of a semigroup $\subseteq$ over $\mathbb{Z}$ is the representation $\rho$ into matrices indexed on $\subseteq$ such that $\rho(x)$ has $y, z$ entry 1 if $y x=z, 0$ otherwise. There is a 1-1 correspondence in general between: (1) representations of $\subseteq$ over $M_{n}(\Re)$ for a commutative semiring $\Re$ with 0,1 ; $(2)$ representations of $\Re(\Im)$ into $M_{n}(\Re)$ (extend linearly); (3) $\subseteq$ actions on an $\Re$ module of the form $\Re \oplus \cdots \oplus \Re$; (4) $\mathfrak{R}(\Im)$ modules which as $\mathfrak{R}$ modules have the form $\mathfrak{R} \oplus \cdots \oplus \mathfrak{R}$.

We will work with the reduced semigroup semiring $\mathfrak{R}_{0}[\Im]$ and the reduced regular representation. The former is the quotient of the semiring by the ideal of elements 
$f$ such that $f(x)=0, x \neq 0$, where $0 \in \subseteq$ is assumed. The latter is the submatrix indexed on $y, z \neq 0$. As usual we can interpret the semigroup semiring as the set of formal sums

$$
\sum a_{s} s, \quad a_{s} \in \mathfrak{R}, s \in \mathfrak{S}
$$

such that $a_{s}=0$ for all but finitely many $s$. The regular representation corresponds to the group ring itself taken as right module.

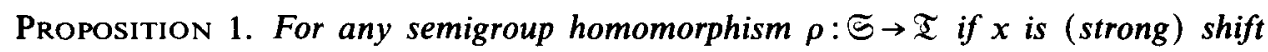
equivalent to $y$ then $\rho(x)$ is to $\rho(y)$.

Proof. If $r s=x, s r=y$ then $\rho(r) \rho(s)=\rho(x), \rho(s) \rho(r)=\rho(y)$. If $r x=x r, x s=s y$, $r s=y^{n}, s r=x^{n}$ then $\rho(r) \rho(x)=\rho(y) \rho(r), \rho(x) \rho(s)=\rho(s) \rho(y), \rho(r) \rho(s)=\rho(y)^{n}$, $\rho(s) \rho(r)=\rho(x)^{n}$.

If $\mathfrak{M}_{1}$ is a proper nonzero submodule of $\mathfrak{M}$ such that $\mathfrak{M}_{1}, \mathfrak{M} / \mathfrak{M}_{1}$ are isomorphic to sums $\mathfrak{A} \oplus \cdots \oplus \mathfrak{R}$ then $\mathfrak{M}_{1}, \mathfrak{M} / \mathfrak{M}_{1}$ give new representations. If no such submodule $\mathfrak{M}_{1}$ exists the representation is called irreducible.

In matrix form this process represents the matrices of the representation as

$$
\left[\begin{array}{ll}
A & 0 \\
B & C
\end{array}\right],
$$

where if we take right modules (actions on row vectors), $A$ corresponds to the invariant submodule $\mathfrak{M}_{1}$ and $C$ to the quotient module $\mathfrak{M} / \mathfrak{M}_{1}$.

Definition. A left (right, 2-sided) ideal in a semigroup $\subseteq$ is a set $\mathscr{I}$ such that for all $r, s \in \Im, x \in \mathscr{I}, s x \in \mathscr{I}(x s \in \mathscr{I}$, all of $x s, s x, s x r \in \mathscr{I})$. The principal left (right, 2-sided ideal) generated by $x$ is $\subseteq x(x \Subset, x \subseteq \cup \Im x \cup \Im x \Subset)$.

Definition. The semigroup $T_{N}$ of partial transformations consists of transformations on subsets of $N^{n}$. Equivalently $T_{n}$ is the semigroup on $n$ square Boolean matrices with at most one 1 per row (acting on zero-one row vectors, each of which represents a subset of $N$ ). $T_{n}^{\mathrm{T}}$ is the semigroup of its transposes, $T_{n} \cap T_{n}^{\mathrm{T}}$ is called the semigroup of partial permutations.

Example. The following are in $T_{n}, T_{n}^{\mathrm{T}}, T_{n} \cap T_{n}^{\mathrm{T}}$

$$
\left[\begin{array}{lll}
1 & 0 & 0 \\
1 & 0 & 0 \\
0 & 0 & 0
\end{array}\right],\left[\begin{array}{lll}
1 & 1 & 0 \\
0 & 0 & 0 \\
0 & 0 & 0
\end{array}\right],\left[\begin{array}{lll}
0 & 0 & 0 \\
1 & 0 & 0 \\
0 & 1 & 0
\end{array}\right] .
$$

Example. The 2-sided ideals in $T_{n}, T_{n} \cap T_{n}^{\mathrm{T}}$ are the set of elements of rank at most $k$ for some $k$ (image size at most $k$ ). The principal left ideals are elements whose image lies in a given set. We multiply as in matrix products in $T_{n}$.

Proposition 2. For any left (right) ideals of $\mathfrak{S}, \mathscr{I}_{1} \subset \mathscr{I}_{2}$, there are left (right) submodules $\mathfrak{R}\left(\mathscr{I}_{1}\right) \subset \mathfrak{A}\left(\mathscr{I}_{2}\right)$ spanned by the corresponding basis elements in the reduced semigroup ring. Each submodule and the quotient are isomorphic to sums $\mathfrak{A} \oplus \cdots \oplus \Re$ as $\mathfrak{R}$-modules. 
Proof. Ideal closure implies the closure needed to be a submodule. The submodules are additively $\Re \oplus \cdots \oplus \Re$ on nonzero elements of $\mathscr{I}_{1}, \mathscr{I}_{2}$, and the quotient is spanned by nonzero elements of $\mathscr{I}_{2} / \mathscr{I}_{1}$.

The relevance of semigroups to shift equivalence of sofic systems comes from the following result of Boyle and Krieger. Let $M_{\infty}\left(\mathbb{Z}^{+}\right)$denote matrices indexed on $\mathbb{Z}^{+}$ with a finite number of nonzero entries each in $\mathbb{Z}^{+}$. We identify $M_{n}\left(\mathbb{Z}^{+}\right)$with a subset of $M_{\infty}\left(\mathbb{Z}^{+}\right)$by $x \rightarrow x \oplus 0$.

THEOREM 3. Topological conjugacy of factor maps is equivalent to strong shift equivalence of their $\lambda$-matrices in the reduced semigroup semiring $\mathbb{Z}_{0}^{+}\left[M_{\infty}\left(\mathbb{Z}^{+}\right)\right]$. For bounded-to-one maps, shift equivalence in this semiring is equivalent to eventual conjugacy.

Two sofic systems are defined to be shift equivalent if they have canonical resolving covers which are shift equivalent.

It is equivalent to work in $M_{\infty}\left(\mathbb{Z}^{+}\right)$or in $M_{n}\left(\mathbb{Z}^{+}\right)$of varying dimensions.

Example. $T_{2} \cap T_{2}^{\mathrm{T}}$ has 6 nonzero elements:

$$
\left[\begin{array}{ll}
1 & 0 \\
0 & 0
\end{array}\right],\left[\begin{array}{ll}
0 & 1 \\
0 & 0
\end{array}\right],\left[\begin{array}{ll}
0 & 0 \\
1 & 0
\end{array}\right],\left[\begin{array}{ll}
0 & 0 \\
0 & 1
\end{array}\right],\left[\begin{array}{ll}
1 & 0 \\
0 & 1
\end{array}\right],\left[\begin{array}{ll}
0 & 1 \\
1 & 0
\end{array}\right] .
$$

Let them be denoted $A_{1}, A_{2}, \ldots, A_{6}$. The right reduced regular representation has the following block structure;

$$
\left[\begin{array}{llllll}
* & * & 0 & 0 & 0 & 0 \\
* & * & 0 & 0 & 0 & 0 \\
0 & 0 & * & * & 0 & 0 \\
0 & 0 & * & * & 0 & 0 \\
* & * & * & * & * & * \\
* & * & * & * & * & *
\end{array}\right] .
$$

Here the $(i, j)$-entry is zero if $A_{i} A_{k} \neq A_{j}$ for every $k$. The $2 \times 2$ main diagonal blocks correspond to quotients in the chain of right ideals $\{0\} \subset\left\{A_{1}, A_{2}, 0\right\} \subset$ $\left\{A_{1}, A_{2}, A_{3}, A_{4}, 0\right\} \subset T_{2} \cap T_{2}^{\mathrm{T}}$.

\section{Decidability}

It happens that for a right resolving factor map given by a $\lambda$-matrix $\sum \lambda_{i} S_{i j}$ each $S_{i}$ has at most one nonzero entry per row, which is 1 . We next show that this means all matrices $R, S$ involved in a shift equivalence of such $\lambda$-matrices can be taken in the smaller semiring $\mathbb{Z}_{0}^{+}\left[T_{\infty}\right]$. Similarly, for left resolving maps it is sufficient to work in $\mathbb{Z}_{0}^{+}\left[T_{\infty}^{\mathrm{T}}\right]$ and for biresolving maps (and thus AFT sofic systems) it is sufficient to work in $\mathbb{Z}_{0}^{+}\left[T_{\infty} \cap T_{\infty}^{\mathrm{T}}\right]$.

For brevity, let $\bar{A}$ denote as mentioned earlier, the actual matrix sum for a formal sum $A$.

THEOREM 4. Let $A, B$ be matrices in the reduced semigroup semirings $\mathfrak{R}_{0}$ of $T_{n}, T_{n}^{\mathrm{T}}$, or $T_{n} \cap T_{n}^{\mathrm{T}}$. If $A, B$ are shift equivalent over the reduced semigroup semiring of $M_{n}\left(Z^{+}\right)$ 
then $A, B$ are shift equivalent over $\Re_{0}$. If $\bar{A}, \bar{B}$ have all row and column sums nonzero, a shift equivalence $R, S$ must lie in $\Re_{0}$. If $A, B$ are strong shift equivalent over $M_{\infty}\left(\mathbb{Z}^{+}\right)$, then they are also over $\mathbb{Z}_{0}^{+}\left(T_{\infty}\right), \mathbb{Z}_{0}^{+}\left(T_{\infty}^{\mathbf{T}}\right)$, or $\mathbb{Z}_{0}^{+}\left(T_{\infty} \cap T_{\infty}^{\mathbf{T}}\right)$.

Proof. Write

$$
R=\sum c_{i} r_{i}, \quad S=\sum d_{i} s_{i}
$$

First assume that for all $i$, row $i$ is nonzero in $\bar{A}$ if and only if column $i$ is nonzero in $\bar{A}$, and row $i$ is nonzero in $\bar{B}$ if and only if column $i$ is nonzero in $\bar{B}$. The same then hold for $\bar{A}^{n}$ and $\bar{B}^{n}$. This means that for all $i$, all terms of $A$ have row $i$ zero if and only if all terms of $A$ have column $i$ zero, and so on.

Let $\Re_{0}=\mathbb{Z}_{0}^{+}\left[T_{n}\right]$. Suppose some row $j$ of $r_{i}$ has two ones.

Case 1. Let $s_{p}$ have $k, j$ entry one. Then $s_{p} r_{i}$ has two ones in $A^{n}$. This is false.

Case 2. Let all $s_{p}$ have column $j$ equal to 0 . Then the product $S R$ is unchanged if row $j$ of $r_{i}$ is replaced by 0 . Also column $j$ of $R S=B^{n}$ thus row $j$ is 0 . So it is unchanged if we replace row $j$ of $r_{i}$ by 0 .

Since $S$ has column $j$ equal to 0 so does $R S=B^{n}$. So then does $B$. And row $j$ of $B$ is zero. Also $R A=B R$ so it has row $j=0$. So changing row $j$ of $R$ to 0 does not affect $R A$. And $B R$ is unchanged since column $j$ of $B$ is 0 .

Therefore we may replace any row of $R, S$ with two ones by 0 , whenever for all $j$ row $j$ is nonzero if and only if column $j$ is nonzero in $A$ and $B$.

This argument also works for the other cases of $\mathfrak{R}_{0}$ when we consider columns.

Next we show that any matrix $A$ is strong shift equivalent over $\mathfrak{R}_{0}$ to one of the desired form $A_{1}$ and that one-step strong shift equivalences in $M_{n}\left(\mathbb{Z}^{+}\right)$from $A$ to $B$ give one-step strong shift equivalences over $M_{n}\left(\mathbb{Z}^{+}\right)$from $A_{1}$ to $B_{1}$.

As $A_{1}$ we take the matrix obtained from $A$ by replacing row and column $j$ by zero whenever $j$ lies on no biinfinite edge sequence of the graph of $\bar{A}$.

For a strong shift equivalence, $A$ to $A_{1}$ we repeat a process of making row $j$ zero whenever column $j$ is zero and vice versa. To do this write $A=A E$ where $E$ is diagonal matrix with $e_{j j}=0, e_{i i}=1, i \neq j$. Refactor as $E A$.

This process continues as long as some vertex of $A$ does not lie in a biinfinite sequence, but is on some edge. But when every vertex on an edge lies on a biinfinite sequence the sets of nonzero rows and columns coincide.

Now $A$ is strong shift equivalent to $A_{1}$ which is obtained by making row and column $j$ zero if $j$ is on no biinfinite edge sequence in the graph of $\bar{A}$.

Consider a strong shift equivalence of one-step $R S=B, S R=A$. Let $R_{1}$ be obtained from $R$ by making a row $j$ zero if $j$ is in no biinfinite edge sequence for $\bar{B}$ and a column $j$ zero if it is in no biinfinite edge sequence for $\bar{A}$. Let $S_{1}$ be obtained from $S$ by the transpose process. Then $R_{1} S_{1} \leq B$ and $R_{1} S_{1}$ has those rows and columns zero which were made zero in $B_{1}$. So $R_{1} S_{1} \leq B_{1}$. Also $S_{1} R_{1} \leq A_{1}$. Let $i_{0} i_{1}$ be a nonzero edge in the graph of $A_{1}$. Then it extends to a biinfinite sequence $\left(i_{n}\right)$.

Since in $R S$

$$
\sum_{k} s_{i_{j} k} r_{k i_{j+1}}=1
$$


we can choose $k_{j}$ so that

$$
s_{i k_{j}} r_{k_{j} i_{j+1}}=1
$$

Then

$$
r_{k_{j} i_{j+1}} s_{i_{j+1} k_{j+1}}=1,
$$

means $\left(k_{j}\right)$ is biinfinite sequence in the graph of $\bar{B}$. So the $k_{j}$ 's are not made zero in $R, S$ and $S_{1} R_{1}$ has $i_{0}, i_{1}$ entry 1 . So $S_{1} R_{1}=A_{1}$, and $R_{1} S_{1}=B_{1}$.

This proves the last statement, since we have over $\mathfrak{M}_{0}$ strong shift equivalences $A$ to $A_{1}, B$ to $B_{1}$ and over $M_{\infty}\left(\mathbb{Z}^{+}\right)$from $A_{1}$ to $B_{1}$, where each term has equal sets of nonzero rows and columns. Then as in the first portion we make the strong shift equivalences over $\mathfrak{R}_{0}$.

Example.

$$
\begin{aligned}
& {\left[\begin{array}{lll}
0 & 0 & 0 \\
0 & 0 & 1 \\
0 & 1 & 0
\end{array}\right]\left[\begin{array}{lll}
1 & 1 & 0 \\
0 & 0 & 1 \\
0 & 0 & 1
\end{array}\right]=\left[\begin{array}{lll}
0 & 0 & 0 \\
0 & 0 & 1 \\
0 & 0 & 1
\end{array}\right],} \\
& {\left[\begin{array}{lll}
1 & 1 & 0 \\
0 & 0 & 1 \\
0 & 0 & 1
\end{array}\right]\left[\begin{array}{lll}
0 & 0 & 0 \\
0 & 0 & 1 \\
0 & 1 & 0
\end{array}\right]=\left[\begin{array}{lll}
0 & 0 & 1 \\
0 & 1 & 0 \\
0 & 1 & 0
\end{array}\right] .}
\end{aligned}
$$

The reduced strong shift equivalence is

$$
\begin{aligned}
& {\left[\begin{array}{lll}
0 & 0 & 0 \\
0 & 0 & 0 \\
0 & 1 & 0
\end{array}\right]\left[\begin{array}{lll}
0 & 0 & 0 \\
0 & 0 & 1 \\
0 & 0 & 0
\end{array}\right]=\left[\begin{array}{lll}
0 & 0 & 0 \\
0 & 0 & 0 \\
0 & 0 & 1
\end{array}\right],} \\
& {\left[\begin{array}{lll}
0 & 0 & 0 \\
0 & 0 & 1 \\
0 & 0 & 0
\end{array}\right]\left[\begin{array}{lll}
0 & 0 & 0 \\
0 & 0 & 0 \\
0 & 1 & 0
\end{array}\right]=\left[\begin{array}{lll}
0 & 0 & 0 \\
0 & 1 & 0 \\
0 & 0 & 0
\end{array}\right] .}
\end{aligned}
$$

THEOREM 5. Shift equivalence of resolving maps (and hence of sofic systems) is decidable.

Proof. It suffices by the previous result to show shift equivalence is decidable in $\mathbb{Z}_{0}^{+}\left[T_{n}\right]$. Let $\left|T_{n}\right|=m+1$ and let $\rho: \mathbb{Z}_{0}^{+}\left[T_{n}\right] \rightarrow M_{n}(\mathbb{Z})$ be the reduced regular representation where we identify a basis $x_{s}$ for $\mathbb{Z}^{m}$ with the nonzero elements $s$ of $T_{n}$.

The module action is right multiplication $x_{s} t=x_{s t}$ if $s t \neq 0, x_{s} t=0$ if $s t=0$. This is the quotient of the regular representation by the 2 -sided ideal generated by zero in $T_{n}$. In matrix form the element $\sum a_{s} s$ goes to the matrix whose $u, v$ entry is the sum of all $a_{s}$ such that $u s=v$. This map embeds $\mathbb{Z}_{0}^{+}\left[T_{n}\right] \rightarrow M_{n}(\mathbb{Z})$ as a subring. If all $a_{s} \geq 0$ then so are the matrix entries which are sums of $a_{s}$.

The $e, s$ entry, where $e \in T_{n}$ is the identity is precisely $a_{s}$. So if it is nonnegative $a_{s} \geq 0$. This proves that $x \in \mathbb{Z}^{+}\left[T_{n}\right]$ if and only if $\rho(x) \geq 0$. Therefore we can apply theorem 10.8 of $[4]$. We have represented $\mathbb{Z}_{0}^{+}\left[T_{n}\right]$ as the nonnegative elements in a finitely additively generated ring of matrices. 
In the next theorem all actions will be on the right. In $T_{n}$ we will take compositions as in matrix products, i.e., $((x) f)=(x)(f g)$.

The origin of the representations in the next theorem is simply any pair of ideals $\mathscr{I}_{k-1} \subset x \Subset+\mathscr{I}_{k-1}$ where $x$ has rank $k$ and $\mathscr{I}_{k-1}$ consists of all elements of rank at most $k-1$. The quotient $\mathbb{Z}^{+}\left(\mathscr{I}_{k-1}+x \subseteq\right) / \mathbb{Z}^{+}\left(\mathscr{I}_{k-1}\right)$ has an action of the symmetric group $s_{k}$ of degree $k$ which permutes elements of the image of $x$ provided we choose an identification of the different size $k$ images. Clifford and Preston [2, Chapter 5] discuss the theory of these representations.

THEOREM 6. For each $k$, there is a representation $\rho$ of $T_{n}$ into matrices over the semigroup semiring $\mathbb{Z}^{+}\left[\mathfrak{s}_{k}\right]$, defined as follows: Let rows and columns be indexed on subsets of size $k$ of $N$. Select a specific isomorphism $h_{s}$ of each subset $S$ of size $k$ to $\{1,2, \ldots, k\}$, e.g., let $h_{s}$ be monotone. The $\underline{x}, \underline{y}$ entry of $\rho(f)$ is $\pi \in \mathfrak{s}_{k}$ if $f(\underline{x})=\underline{y}$ as sets and as ordered sets $h_{x} \pi=f h_{y}$. If $f(\underline{x}) \neq y$ the entry is zero. This representation (taken into $M_{\infty}\left(\mathbb{Z}^{+}\left(s_{k}\right)\right)$ ) is compatible with the inclusion $T_{n} \subset T_{n+1}$. Each of these representations yield representations of $\mathbb{Z}_{0}\left[T_{n}\right]$ which are onto, even restricted to $\mathbb{Z}_{0}\left[T_{n} \cap T_{n}^{\mathrm{T}}\right]$, and the direct sum of these representations is onto.

Proof. Consider $\underline{x}, \underline{w}$ entry of $\rho(f g)$. It is nonzero if and only if $(\underline{x}) f g=\underline{w}$ as sets. This is if and only if for some $\underline{y},(\underline{x}) f=\underline{y},(\underline{y}) g=\underline{w}$, i.e., if and only if the product of the matrices for $\rho(f), \rho(g)$ has nonzero $\underline{x}, \underline{y}$ entry.

If it is nonzero, the set $\underline{y}$ will be unique. The product of $\rho(f) \rho(g)$ has $\underline{x}, \underline{w}$ entry

$$
h_{\underline{x}}^{-1} f h_{\underline{y}}\left(h_{\underline{y}}^{-1} g h_{\underline{w}}\right)=h_{\underline{x}}^{-1} f g h_{\underline{w}},
$$

the $\underline{x}, \underline{w}$ entry of $\rho(g)$. So we have a representation.

If we include $T_{n}$ in $T_{n+1}$ and extend the family of sets $\underline{x}, \underline{y}$ and of mappings $h_{s}$ then the nonzero entries of $\rho(f), f \in T_{n}$ will still lie only in the set of ordered pairs $\underline{x}, \underline{y}$ of original entries since the domain and range of $f$ both lie in $\{1,2, \ldots, n\}$. And the representation gives the same result on both. The mapping is onto since we can choose for all $\underline{x}, \underline{y}, \pi$ a partial permutation of rank $k$ mapping $\underline{x}$ to $\underline{y}$ with effect corresponding to $\pi$ multiplication. This partial permutation will not be defined on any other size $k$ subset, so this is the only nonzero entry.

Corollary 7. Strong shift and shift equivalence classes of the images of these representations are strong shift and shift equivalence invariants.

COROLlary 8. If we compose these representations with any rational irreducible representation the rational group ring of the symmetric group, they are irreducible.

Proof. An irreducible representation of $\mathbb{Q}\left(s_{k}\right)$ is onto a full matrix algebra over $\mathbf{Q}$ so since those are onto matrices over $\mathbb{Q}\left(s_{k}\right)$ the composite representation is a full matrix ring over $\mathbb{Q}$. It therefore cannot be reduced.

Proposition 9. The sum of the representations given is an isomorphism on $\mathbb{Z}\left(T_{n} \cap T_{n}^{\mathrm{T}}\right)$. Proof. By counting dimensions this follows from the onto result. Rank $k$ partial permutations on $N$ number $k !\left(\begin{array}{l}n \\ k\end{array}\right)^{2}$ by choice of permutation, domain, image set. 
The $k$ th representation is onto $\left(\begin{array}{l}n \\ k\end{array}\right) \times\left(\begin{array}{l}n \\ k\end{array}\right)$ matrices over the group ring which has $k$ ! elements, the image dimension also is $k !\left(\begin{array}{l}n \\ k\end{array}\right)^{2}$.

\section{Example}

To illustrate this procedure, we give an example of a sofic system not conjugate to its inverse, but whose domain is conjugate to its inverse.

The periodic data of a shift and its transpose are the same. M. Boyle has remarked that the subshift of points with preimage having more than one point provides an alternative way to distinguish this shift from its transpose. The example will be almost of finite type so we can work in $T_{n} \cap T_{n}^{\mathrm{T}}$.

The degree 1 representation of Theorem 6 sends an element $A$ to $\bar{A}$, the sum of the matrices whose formal sum is $A$. Each transformation is represented by itself. The element $\bar{A}$ is the domain subshift.

The rank 2 representation of a rank 2 partial permutation $g$ will have a single nonzero entry. If we take as sets $\{2,3\},\{1,3\},\{1,2\}$ in the order of their complements $\{1\},\{2\},\{3\}$ the nonzero entry is located in that row where $g$ has no ones and in that column where $g$ has no ones.

We take a formal sum of rank 2 partial permutations whose image has nonzero entries located where the ones occur in this Boolean matrix

$$
\left[\begin{array}{lll}
1 & 0 & 0 \\
1 & 1 & 0 \\
1 & 0 & 1
\end{array}\right]
$$

For example, we take this formal sum of matrices;

$$
X=\left[\begin{array}{lll}
0 & 0 & 0 \\
0 & 1 & 0 \\
0 & 0 & 1
\end{array}\right]+\left[\begin{array}{lll}
1 & 0 & 0 \\
0 & 0 & 0 \\
0 & 0 & 1
\end{array}\right]+\left[\begin{array}{lll}
0 & 0 & 0 \\
0 & 1 & 0 \\
0 & 0 & 1
\end{array}\right]+\left[\begin{array}{lll}
0 & 1 & 0 \\
0 & 0 & 0 \\
0 & 0 & 1
\end{array}\right]+\left[\begin{array}{lll}
0 & 1 & 0 \\
0 & 0 & 1 \\
0 & 0 & 0
\end{array}\right]
$$

Let the functions $h$ be monotone. Then the group ring images of the respective terms are

$$
\left[\begin{array}{lll}
1 & 0 & 0 \\
0 & 0 & 0 \\
0 & 0 & 0
\end{array}\right],\left[\begin{array}{lll}
0 & 0 & 0 \\
0 & 1 & 0 \\
0 & 0 & 0
\end{array}\right],\left[\begin{array}{lll}
1 & 0 & 0 \\
0 & 0 & 0 \\
0 & 0 & 0
\end{array}\right],\left[\begin{array}{lll}
0 & 0 & 0 \\
1 & 0 & 0 \\
0 & 0 & 0
\end{array}\right],\left[\begin{array}{lll}
0 & 0 & 0 \\
0 & 0 & 0 \\
1 & 0 & 0
\end{array}\right] .
$$

Then if we map the group semiring $\mathbb{Z}^{+}\left(\mathbb{Z}_{2}\right)$ into the Boolean algebra by $\mathbb{Z}_{2} \rightarrow\{1\}$, $0 \rightarrow 0$, we obtain

$$
\left[\begin{array}{lll}
1 & 0 & 0 \\
1 & 1 & 0 \\
1 & 0 & 1
\end{array}\right]
$$

This matrix is not shift equivalent to its transpose (which is the image of the transpose formal sum). 
Now to make the domain symmetric we just add rank 1 partial permutations.

$$
Y=\left[\begin{array}{lll}
0 & 0 & 0 \\
1 & 0 & 0 \\
0 & 0 & 0
\end{array}\right]+\left[\begin{array}{lll}
0 & 0 & 0 \\
1 & 0 & 0 \\
0 & 0 & 0
\end{array}\right]+\left[\begin{array}{lll}
0 & 0 & 0 \\
0 & 0 & 0 \\
0 & 1 & 0
\end{array}\right] .
$$

Now $X+Y$ is the required example. The sum $\bar{X}+\bar{Y}$ is symmetric.

\section{Rational shift equivalence of sofic shifts}

THEOREM 10. Any function from $\mathbb{Z}_{0}\left[T_{\infty}\right]$ to an abelian group satisfying $f(x y)=f(y x)$ and $f(x+y)=f(x)+f(y)$ is (i) a linear function composed with the function assigning to $\sum n_{i} y_{i}$ the sum $\sum n_{i} z_{i}$ where $z_{i}$ is the strong shift equivalence class of $y_{i}$ in $T_{\infty}(\mathbb{Z})$; (ii) a linear function of the traces of representations of Theorem 6 to $M_{n}\left(\mathbb{Z}\left(\mathfrak{s}_{k}\right)\right.$ ) composed with representations of the symmetric group.

Proof. Any such function factors through the abelian group obtained from $\mathbb{Z}_{0}\left[T_{\infty}\right]$ by identifying $x y$ with $y x$. But this is precisely the group of $\sum n_{j} z_{i}$.

We next show that strong shift equivalence classes of partial transformations $\tau$ are conjugacy classes of their final permutations. The final permutation is the partial transformation induced by $\tau$ on

$$
D=\bigcap_{n=1}^{\infty} \tau^{n}\left(\mathbb{Z}^{+} \backslash\{0\}\right) .
$$

These sets converge since $\tau$ has finite image and they are decreasing. The mapping $\tau$ restricted to $D$ will be onto since $\tau(D)$ is also the image of $\tau^{n+1}, n$ large. So $\tau$ is a permutation of $D$. Let $\tau^{\prime}$ be this partial permutation.

To obtain a strong shift equivalence of $\tau$ to $\tau^{\prime}$, let $e_{n}$ be an idempotent with image $\tau^{n}(D)$. We can write $\tau$ restricted to $\tau^{r}(D)$ as $\tau e_{r+1}$. And $e_{r+1} \tau$ is $\tau$ restricted to $\tau^{r+1}(D)$. Here the domain of $e_{n}$ is assumed to equal its image.

Strong shift equivalences will not alter the traces under any representation. Partial permutations such as $\tau$ which have the same image as $\tau^{2}$ are conjugate if and only if their cycles have the same sizes. And if they are strong shift equivalent their cycles must have the same sizes, since strong shift equivalences will be conjugacies on their domains. Let $\theta$ be the mapping assigning to $\sum n_{i} z_{i}$ their traces under all the representations to $M_{n}\left(s_{k}\right)$ of Theorem 6 composed with irreducible representations of the symmetric group. Suppose $\theta$ has a nonzero element $x$ in its kernel. Assume $x$ is a linear combination of partial permutations of types $\tau$ in distinct conjugacy classes. Let $k$ be the largest rank of partial permutations in $x$. Then partial permutations of rank less than $k$ go to zero.

Assume the representative partial permutations all fix $\underline{k}=\{1,2, \ldots, k\}$. Then their matrices have only the $\underline{k}, \underline{k}$ entry nonzero and it will be a permutation from a conjugacy class. The trace will be that of the permutation. But the traces of irreducible representations of any finite group are linearly independent on classes by orthogonality relations.

COROLLARY 11. The above functions are invariants of shift equivalence in $\mathbb{Z}_{0}\left[T_{\infty}\right]$ and $Q_{0}\left[T_{\infty}\right]$. 
Proof. The traces of matrices over $\mathbb{Q}$ are shift equivalence invariants.

There exists a semigroup homomorphism $\mathbb{Q}_{0}\left(T_{n}\right) \rightarrow \mathbb{Q}_{0}\left(T_{n} \cap T_{n}^{\mathrm{T}}\right)$. Take the map $\pi_{1}$ of $Q_{0}\left(T_{n}\right)$ onto the direct sum of matrix rings of group rings defined by Theorem 6. Compose this with the inverse of the isomorphism $\pi_{2}$ of $\mathbb{Q}_{0}\left(T_{n} \cap T_{n}^{\mathrm{T}}\right)$ onto the same image of Proposition 9. The inclusion gives a right inverse $\lambda_{1}: \mathbb{Q}_{0}\left(T_{n} \cap T_{n}^{\mathrm{T}}\right) \rightarrow$ $\mathbf{Q}_{0}\left(T_{n}\right)$. The kernel of $\pi_{1}$ can be shown to consist of nilpotent elements by the last part of the proof of the next theorem or because in the regular representation of the group ring these elements will be zero on and above the main diagonal.

Two major questions which remain are, what algebraic invariants can be computed from $\mathbb{Z}_{0}^{+}\left[T_{n}\right]$ and is there any difference between shift and strong shift equivalence in this setting? Regarding the former, the characteristic polynomials of all representations give rational invariants closely related to periodic data [1]. Over integral group rings shift equivalence is in principle decided by [4]. However, we do not know whether strong shift equivalence in integral group rings coincides with shift equivalence in even the simplest case of the group $\mathbb{Z}_{2}$.

Over the full ring $\mathbb{Z}_{0}\left[T_{n}\right]$ there will exist additional shift equivalence invariants beyond what the representations give. The kernel of all representations is a nilpotent ideal. The additional invariants have Jordan form type but their structure depends on the nature of the image in the representations especially on eigenvalue multiplicity. This structure exists over fields $\mathbb{F}$ in $\mathbb{F}_{0}\left[T_{n}\right]$. We are not able to solve completely the question of whether strong shift equivalence coincides with shift equivalence over $\mathbb{Q}_{0}\left[T_{n}\right]$ but the next theorem represents results concerning these topics.

THEOREM 12. For any finite dimensional algebra $\mathscr{A}$ over a field $\mathbb{F}$ for all $x$ there exists a polynomial $e(x)$ which is idempotent such that for all large $n$ right multiplication by $e(x)$ is an isomorphism on the ideal generated by $x^{n}$.

If $x, y$ are shift equivalent they have the same minimum polynomial up to powers of $x$. This means we can take a common polynomial $e(x)$. Then $x, y$ are shift equivalent if and only if separately $x e(x), y e(y)$, and $x-x e(x), y-y e(x)$ are shift equivalent. The elements $x e(x), y e(y)$ are always shift equivalent if and only if they are strong shift equivalent and the elements $x-x e(x), y-y e(y)$ are always shift equivalent to 0 and nilpotent. For nilpotent elements in $\mathrm{F}_{0}\left[T_{n}\right]$ shift equivalence coincides with strong shift equivalence if and only if the characteristic of $\mathbb{F}$ does not divide $n !$. Let $\pi_{1}$ denote the representation of $\mathbb{F}_{0}\left[T_{n}\right]$ into the direct sum of matrix rings over $\mathbb{F}\left[\mathfrak{s}_{k}\right]$ obtained from the representations of Theorem 6 , by tensor product with $\mathbb{F}$. The kernel of $\pi_{1}$ is a 2-sided ideal of nilpotent elements.

Proof. We decompose an element $x$ as a sum of nilpotent and invertible summands in a finite dimensional $\mathbb{F}$ algebra $\mathscr{A}$. The left ideals $x^{n}$ of $\mathscr{A}$ must eventually become constant, since their dimensions are nonincreasing. Choose an $x^{n}$ to represent such an ideal.

To find $e(x)$ we let $p(x)$ be the minimum polynomial of $x$. Let $p(x)=x^{q} p_{1}(x)$, $\left(x, p_{1}(x)\right)=1$. Let

$$
e(x)=x^{m} r(x),(r(x), x p(x))=1, \quad m \geq q, n, 1 .
$$


Then idempotence of $e(x)$ is equivalent to

$$
p(x) \mid e(x)^{2}-e(x) \text { or } p_{1}(x) \mid x^{m} r(x)-1 \text {. }
$$

Since $\left(p_{1}(x), x^{m}\right)=1$, we can find $r(x)$. From $p(x) \mid\left(x^{m} r(x)-1\right)$ follows the property that $e(x)$ generates the ideal generated by $x^{n}$, since in the ring $x^{2 m} r(x)=x^{m}$.

Then $e(x)$ depends only the minimum polynomial satisfied by $x$. If $p(x)=0, r, s$ are a shift equivalence then $r p(x) s=0$ is $y^{n} p(y)$ for some $n$. So they have the same minimum polynomial up to powers of $x$.

Given one-step strong shift equivalence $r s=y, s r=x$, then $e(x) s=\operatorname{se}(y), r e(x)=$ $e(y) r$ and $e(x) r s e(x)=e(x) x, \operatorname{se}(x) e(x) r=e(y) y$. Also

$$
\begin{aligned}
& (1-e(x)) r s(1-e(x))=(1-e(x)) x, \\
& r(1-e(x))(1-e(x)) s=(1-e(y)) y .
\end{aligned}
$$

So we have strong shift equivalences between their two parts. This also goes through for shift equivalences.

If we have shift equivalences of $\operatorname{lag} n, r_{1}, s_{1}$ from $x e(x)$ to $y e(y)$ and $r_{2}, s_{2}$ of lag $n$ from $x-x e(x)$ to $y-y e(y)$ we can assume $r_{1}, s_{1}$ are divisible by $x e(x)$ and $r_{2}, s_{2}$ by $x-x e(x)$ possibly increasing lag. Then $r_{1} s_{2}, r_{2} s_{1}, s_{2} r_{1}, s_{1} r_{2}$ all have factors $e(x)(1-e(x))$ and are zero. Then $r_{1}+r_{2}, s_{1}+s_{2}$ give shift equivalences $x$ to $y$. (This is true for strong shift equivalence if $r_{1}=r_{1} e(x), s_{1}=e(x) s_{1}, r_{2}=r_{2}-r_{2} e(x)$, $s_{2}=s_{2}-e(x) s_{2}$.)

Suppose we have a shift equivalence $r, s$ on the invertible parts $x e(x), y e(y)$. Choose a polynomial $f(x)$ with $x f(x) e(x)=e(x)$, since $x \mid e(x)$. Let $r, s$ be divisible by $x e(x)$. Then if $r s=y^{n} e(y), s r=x^{n} e(x), r(f(x) e(x))^{n}$ and $s$ give a strong shift equivalence since for instance

$$
r(f(x) e(x))^{n} s=f(y)^{n} e(y)^{n} r s=f(y)^{n} e(y) y^{n} e(y)=e(y) .
$$

Therefore on the invertible parts shift equivalence and strong shift equivalence coincide.

If the characteristic $\mathfrak{p}$ of $\mathbb{F}$ divides $n$ ! then we will show shift equivalence and strong shift equivalence differ in $\mathbb{F}\left(\mathfrak{s}_{n}\right)$. By the maps to and from $\mathbb{F}\left(T_{n}\right)$ this implies they differ in $\mathbb{F}\left(T_{n}\right)$. Strong shift equivalence preserves the linear (not multiplicative) map on $\mathbb{F}\left[s_{n}\right]$ which identifies two elements in the same class (by the proof of Theorem 10). Under this $(1-x)$ does not go to zero since they are in different conjugacy classes, if $x$ has order $p$ in $s_{n}$. But

$$
(1-x)^{p}=1-x^{p}=0,
$$

so $(1-x)$ is nilpotent and shift equivalent to zero. To complete the proof we show nilpotent elements in $\mathbb{F}\left(T_{n}\right)$ are strong shift equivalent to zero.

Here we use the representations into group rings and that they give an isomorphism on $T_{n} \cap T_{n}^{\mathrm{T}}$.

Let $x$ be nilpotent. Suppose the highest rank occurring in $x$ is $k$. We claim there exists a sum $e_{k}$ of partial permutations of rank at most $k$ which is the identity on all partial transformations of rank at most $k$, on the right. 
To obtain $e_{k}$ use the fact that the sum of the representations to matrix groups in 1-1 onto on $\mathbb{F}\left(T_{n} \cap T_{n}^{\mathrm{T}}\right)$. Then take the identity element in all summands for $k_{i} \leq k$. It can be obtained as an integral sum of partial permutations of rank at most $k$, by the previous construction. This has the desired property on all partial permutations. But all rank $k_{1}$ transformations $f$ can be written $g h$ when $h$ is a rank $k_{1}$ partial permutation with the same image, defined on a set mapping 1-1 onto to the image. So $e_{k}$ has been found. Then $x=x e_{k}$ is shift equivalent to $e_{k} x$. This means that now among rank $k$ elements of $x$ we have only partial permutations. Moreover if $x$ is any element in the kernel of $\pi_{1}$ repeated refactoring $x e_{k}$ to $e_{k} x$ reduces $x$ to zero. Therefore, if $x \in \operatorname{ker}\left(\pi_{1}\right)$ it is nilpotent. The kernel is a 2 -sided ideal consisting solely of nilpotent elements so is a nilpotent ideal.

Let $\pi_{1}(x)=\left(0, \ldots, 0, x_{k}, \ldots, x_{1}\right)$ in the direct sum of matrices over group rings. Let $f_{k}=(0, \ldots, \mathscr{I}, 0, \ldots, 0)$ with the $\Phi$ in degree $k$.

We may write $x=x_{1}+x_{2}$ where $x_{2}$ consists of rank $k$ partial permutations, $x_{1}$ of rank less than $k$ partial transformations. Then $\pi_{2}^{-1} f_{k}$ is zero on all partial permutations of rank less than $k$. So $g \lambda_{1} \pi_{2}^{-1} f_{k}$ is zero for partial transformations $f$ of rank less than $k$ since $f=g h$ as above. Let $\phi=\lambda_{1} \pi_{2}^{-1} f_{k}$. Then $x \phi=x_{1} \phi=\phi x_{1} \phi$ since $\phi$ is central in image $\pi_{1}$, so in $\mathbb{F}\left(T_{n} \cap T_{n}^{\mathrm{T}}\right)$. We have $x(1-\phi)$ consists of terms of rank at most $(k-1)$. Let $\pi_{k}$ be the representation to $M\left(s_{k}\right)$. Let $\pi_{k}(x)=x_{3}$. Now we can extend any strong shift equivalence on $x_{3}$ to $x$. Let $x \phi=s r$ where $r, s$ are divisible by $\phi$ on each side. Then

$$
(s+x(1-\phi))(r+(1-\phi))=s r+x-x \phi=x .
$$

So if we reverse this factorization we extend a strong shift equivalence on $x_{3}$. So we can make $x \phi$ zero if $x_{3}$ is strong shift equivalent to 0 .

Since the characteristic of $\mathbb{F}$ does not divide the order of $\mathfrak{s}_{k}$ the rings $\mathbb{F}\left[\mathfrak{s}_{k}\right]$ are direct sums of matrix rings over division algebras and in them nilpotents are strong shift equivalent to zero. This means we can make all parts of $x$ of rank at most $k-1$. By induction $x$ is strong shift equivalent to zero (strong shift equivalence preserves nilpotency).

The study of strong shift equivalence in $\mathbb{F}\left(T_{n}\right)$ is a problem of considerable interest. Is it always the same as shift equivalence together with whatever characteristic polynomials can be defined over $\mathbb{F}$ ?

Acknowledgement. The authors would like to acknowledge the help of Mike Boyle in clarifying our proofs, suggesting an example such as we have given, and comments leading to additional theorems.

\section{REFERENCES}

[1] M. Boyle \& W. Krieger. Almost Markov and shift equivalent sofic systems. Dynamical Systems (Proceedings, University of Maryland 1986-87), ed. J. C. Alexander, Springer: New York, 1988, pp. 33-93.

[2] A. Clifford \& G. Preston. The Algebraic Theory of Semigroups. Amer. Math.: Providence, R.I., 1961. 
[3] T. Hamachi \& M. Nasu. Topological conjugacy for 1-block factor maps of subshifts and sofic covers. Dynamical Systems (Proceedings, University of Maryland 1986-87), ed. J. C. Alexander, Springer: New York, 1988. pp. 251-260.

[4] K. H. Kim \& F. W. Roush. Decidability of shift equivalence. Dynamical Systems (Proceedings, University of Maryland 1986-87), ed. J. C. Alexander, Springer: New York, 1988. pp. 374-424.

[5] M. Nasu. Topological conjugacy for sofic systems. Ergod. Th. \& Dynam. Sys. 6 (1986), 265-280. 